\title{
Macroscopic tunnel splittings in superconducting phase qubits
}

\author{
Philip R. Johnson * William T. Parsons, Frederick W. Strauch甘 \\ J.R. Anderson, Alex J. Dragt, C.J. Lobb, and F.C. Wellstood \\ Department of Physics, University of Maryland, College Park, MD 20850
}

(Dated: October 23, 2018)

\begin{abstract}
Prototype Josephson-junction based qubit coherence times are too short for quantum computing. Recent experiments probing superconducting phase qubits have revealed previously unseen fine splittings in the transition energy spectra. These splittings have been attributed to new microscopic degrees of freedom (microresonators), a previously unknown source of decoherence. We show that macroscopic resonant tunneling in the extremely asymmetric double well potential of the phase qubit can have observational consequences that are strikingly similar to the observed data.

PACS numbers: 74.50.+r, 03.67.Lx, 85.25.Cp, 03.65.Xp
\end{abstract}

Recent experiments by Simmonds et al. [1] and Cooper et al. 2] reveal previously unseen fine splittings in the transition energy spectra of superconducting phase qubits. These splittings are interpreted as resulting from coupling between the circuit's collective dynamical variable (the superconducting phase describing the coherent motion of a macroscopic number of Cooper pairs) and microscopic two-level resonators, hereafter called microresonators, within Josephson tunnel junctions. Microresonators may be an important decoherence mechanism 1, 2, 3] for many different superconducting qubit devices [4, 5, 6] with broader implications for Josephson junction physics generally. Key questions remain however. Are all of the observed splittings truly a microscopic property of junctions? Could they instead be a macroscopic property of the particular circuit, or a combination of microscopic and macroscopic phenomena?

In fact, macroscopic resonant tunneling (MRT) can produce spectral splittings in multiwell systems by lifting degeneracies between the states of different wells. These effects have been probed by Rouse et al., Friedman et al., and others [7] in superconducting circuits involving asymmetric double wells with a few left well states, and $\lesssim$ 10 right-well states. MRT effects have also been demonstrated by Crankshaw et al. [8] in three-junction flux qubits, another system in which spurious splittings have been reported [9]. What is not obvious is that MRT effects can be important for systems with extremely asymmetric double well potentials, like the rf SQUID phase qubit [1, 2], that have hundreds or thousands of right well states. In this Letter, we analyze the phase qubit in this limit and show that MRT produces surprisingly complex observational consequences that are strikingly similar to some of the observed data 1, 2]. MRT is therefore a possible mechanism for fine splittings in a phase qubit and requires further examination.

Figure 1(a) shows the circuit schematic for an rf SQUID. The device is a superconducting loop of inductance $L$ interrupted by a single Josephson junction with capacitance $C$ and critical current $I_{c}$, inductively coupled
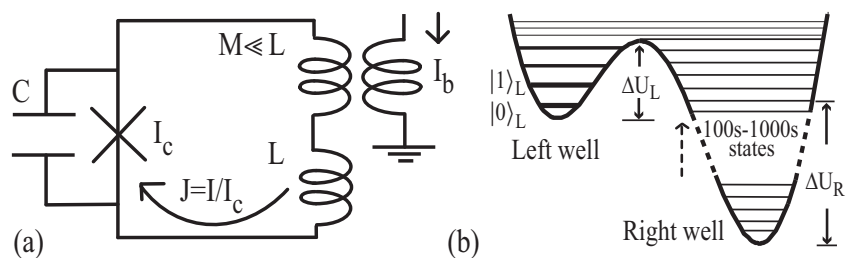

FIG. 1: (a) Circuit diagram for an rf SQUID phase qubit. (b) The device can be tuned via an inductively coupled bias line to give an extremely asymmetric double-well.

to a flux-bias line. The circuit Hamiltonian is

$$
H=4 E_{C} p^{2} / \hbar^{2}+E_{J}\left(\gamma^{2} / 2 \beta-\cos \gamma-J \gamma\right)
$$

where $\gamma$ is the gauge invariant phase difference across the junction, $p=\hbar Q / 2 e$ is the momentum conjugate to $\gamma(Q$ is the charge on the plates of the capacitor), $\beta=$ $2 \pi I_{c} L / \Phi_{0}$ is the modulation parameter $\left(\Phi_{0}=h / 2 e\right.$ is the flux quantum), and $J=I / I_{c}$ is the dimensionless current that is induced in the loop by the applied flux bias. The charging energy $E_{C}=e^{2} / 2 C$ and Josephson energy $E_{J}=$ $I_{c} \Phi_{0} / 2 \pi$ determine the regime of superconducting qubit behavior; for a phase qubit $E_{J} \gg E_{C}$.

The shape of the circuit's potential energy function $U(\gamma)$ depends on $\beta$ and the bias $J$. For $\beta \lesssim 3 \pi / 2$, it is possible to bias the circuit so that the potential has the highly asymmetric double-well shape shown in Fig. 1(b), tuned to give a shallow upper left well with just a few left-localized states, denoted by $|n\rangle_{L}$, and a deep right well with many right-localized states, denoted by $|m\rangle_{R}$. Simmonds et al. 1] -motivated by a number of attractive features including reduced quasiparticle generation, tunable anharmoniticity of the left well potential, inductive isolation from and reduced sensitivity to bias noise, and nice read-out properties-have proposed using the $\mathrm{rf}$ SQUID with an extremely asymmetric double well potential as a phase qubit [4].

Making a cubic approximation to the left well, we de- 


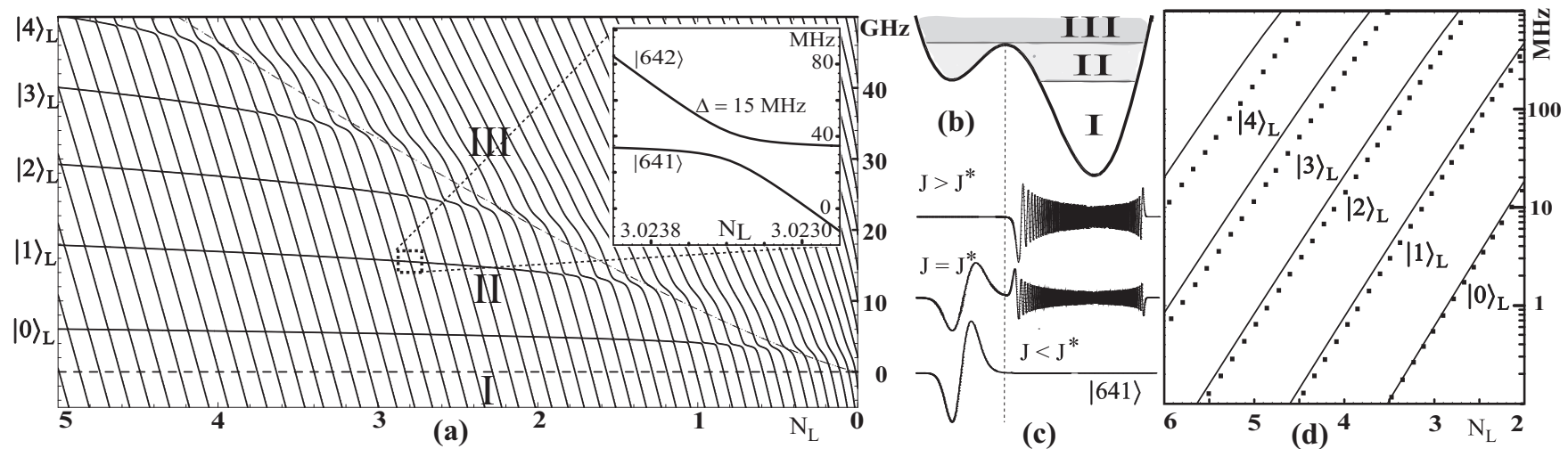

FIG. 2: (a) Numerically computed spectrum of phase qubit when $I_{c}=8.531 \mu \mathrm{A}, C=1.2 \mathrm{pF}$, and $L=168 \mathrm{pH}(\beta=4.355)$. Energies are plotted in units of frequency. The inset shows the avoided crossing due to resonant tunnel coupling between the left well state $|1\rangle_{L}$ and a highly excited right well state. (b) The circuit parameters give an asymmetric double well like that shown. (c) Wavefunctions of the $k=641$ eigenstate for bias values near the avoided crossing shown in the inset. (d) Solid points are numerically computed sizes and locations of the splittings. Solid lines are splitting sizes derived from WKB theory.

rive the plasma frequency for small oscillations

$$
\omega_{L}=\omega_{0}\left(1-\beta^{-2}\right)^{1 / 8}\left[2\left(J^{*}-J\right)\right]^{1 / 4},
$$

where $\omega_{0}=\sqrt{8 E_{c} E_{J} / \hbar^{2}}$, and

$$
J^{*}=\left(1-\beta^{-2}\right)^{1 / 2}+\beta^{-1} \arccos \left(-\beta^{-1}\right)>1
$$

is the critical bias for which the left well vanishes. Note that the effective critical current is $I^{*}=I_{c} J^{*}>I_{c}$. The approximate number of left-well states is

$$
N_{L}=\frac{\Delta U_{L}}{\hbar \omega_{L}} \simeq \frac{2^{3 / 4}}{3} \sqrt{\frac{E_{J}}{E_{C}}}\left(1-\beta^{-2}\right)^{-3 / 8}\left(J^{*}-J\right)^{5 / 4},
$$

where $\Delta U_{L}$ is the barrier height. The level spacing in the right well is approximately $\hbar \omega_{R}$, where $\omega_{R}$ is the right well plasma frequency, and the number of right well states is approximately $N_{R} \simeq \Delta U_{R} / \hbar \omega_{R}$, where $\Delta U_{R}$ is the depth of the right well.

Figure 2(a) shows the energy spectrum as $J$ is varied for $0 \leq N_{L} \leq 6$ and $C=1.2 \mathrm{pF}, L=168 \mathrm{pH}$, and $I_{c}=8.531 \mu \mathrm{A}$, giving $\beta=4.355, I^{*}=11.659 \mu \mathrm{A}$, and $\omega_{L} / 2 \pi \sim 10 \mathrm{GHz}$. These are the circuit parameters from 1], assuming that the critical current quoted there is $I^{*}$. To obtain the energy spectrum we diagonalize the Hamiltonian in Eq. (11) using a discrete Fourier grid representation [10], thereby obtaining a numerical solution for the eigenvalues $E_{k}(J)$ and eigenstates $|k(J)\rangle$ of the full double-well system versus the bias $J$. A harmonic approximation to the right well yields approximately 500 states below the left well; the full calculation yields $N_{R} \simeq 600-700$ states, depending on the bias 11 .

In Fig. 2(a) we define the zero of energy to be at the bottom of the left well. We note two different types of energy levels: horizontal $(H)$ branches and near vertical $(V)$ branches. From our definition of zero energy, eigenvalues corresponding to states mainly localized in the right well [region I of Figs. 2(a) and (b)] fall with increasing $J$, and are thus nearly vertical. The energy levels in region III correspond to delocalized states fully above the left well. The dashed line in Fig. 2(a) dividing regions II and III indicates the energy at the top of the left-well barrier. In region II, eigenstates whose energies lie along $H$ branches are primarily localized in the left well $(H \sim L)$. The number of left-well states at bias $J$ is consistent with $N_{L}$ from Eq. (41). Eigenstates whose energies lie along $V$ branches are primarily localized in the right well $(V \sim R)$. Their energies fall at essentially the rate of the falling right well. Note that in Fig. 2(a) the density of right-well states is comparable to that of the left-well, despite $N_{R} \gg N_{L}$.

Every apparent intersection of an $H$ and $V$ energy level in Fig. 2(a) is an avoided crossing (see inset). Degeneracies are lifted by resonant tunneling of left-well states $|n\rangle_{L}$ and right-well states $|m\rangle_{R}$. Left of an avoided crossing between $k$ and $k+1$ eigenstates we find that $|k\rangle \cong|n\rangle_{L}$ and $|k+1\rangle \cong|m\rangle_{R}$. Right of the crossing the states swap, becoming $|k\rangle \cong|m\rangle_{R}$ and $|k+1\rangle \simeq|n\rangle_{L}$. At the avoided crossing $|k\rangle \cong\left(|n\rangle_{L}+|m\rangle_{R}\right) / \sqrt{2}$ and $|k+1\rangle \cong\left(|n\rangle_{L}-|m\rangle_{R}\right) / \sqrt{2}$. Figure 2(c) shows the wavefunctions for the $k=641$ eigenstate before, at, and after the splitting shown in the inset in Fig. 2 (a). The distribution of splitting magnitudes along the first five energy branches are plotted in Fig. 2(d) as solid points. Gaps larger than $1 \mathrm{MHz}$ are within the resolution of recent experiments. Along each left-well energy branch the tunnel splittings are regularly spaced with magnitudes that decrease exponentially with $N_{L}$. We have numerically computed spectra for a variety of circuit parameters, including $I_{c}=2 \mu \mathrm{A}$ and $C=0.5 \mathrm{pF}$ which are comparable to those reported in 2]. In each case the spectrum looks qualitatively similar to Fig. 2 (a). We note that the predicted gap sizes are strikingly similar to those reported in 1, 2] $(\sim 1-100 \mathrm{MHz})$. 
The complex collection of energy splittings has both direct and indirect effects that should be taken into account when analyzing the experimental data. Consider a double frequency microwave spectroscopic method, like that used in [1]. Microwaves of frequency $\omega_{01}$ are applied to drive the $0 \rightarrow 1$ transition. Excitation of the $|1\rangle_{L}$ state is detected with a measurement microwave pulse of frequency $\omega_{13}$, which drives the $1 \rightarrow 3$ transition. The $|3\rangle_{L}$ state's exponentially greater amplitude to be found in the right well compared to the $|0\rangle_{L}$ and $|1\rangle_{L}$ states allows an adjacent detection SQUID to easily detect the change in the qubit's flux. This method directly probes splittings along many of the energy branches shown in Fig. 2(d). Cooper et al. have introduced a new spectroscopic technique that can probe deeper left wells where $N_{L}>4$. This method applies a few-nanosecond current pulse changing the bias so that $N_{L} \gtrsim 2$ by briefly tilting the potential adiabatically with respect to the left well period $T_{L} \equiv 2 \pi / \omega_{L} \sim 100 \mathrm{ps}$ [2]. Since the measurement pulse moves left-well states to the right along horizontal $(H)$ energy branches [see Fig. 2(a)], read-out should be influenced by the exponentially larger splittings present for smaller $N_{L}$. For example, the measurement pulse may move a deep well state to one of the large splitting degeneracies near $N_{L} \sim 2$, whose presence may produce a significant perturbation on read-out fidelity. Thus the current pulse method is also sensitive to large splittings along multiple energy branches.

MRT degeneracies also have very narrow bias value widths. For example, the inset of Fig. 2(a) shows a splitting width of less than $0.1 \mathrm{nA}$. The bias widths become only smaller for splittings at larger $N_{L}$. The horizontal axis of Fig. 2(a) corresponds to more than 300 nA. Typical experiments sampling only a limited number of bias values likely probe only a subset of the (many) MRT splittings. Changes in experimental conditions (e.g. bias drift and noise, or temperature cycling) may generate surprisingly large shifts in the observed splitting distributions if they result in a different subset of sampled MRT degeneracies. These or other features could result in transition spectra with a varying distribution of splitting sizes and bias-value locations which, due to their complexity and variability in time, might appear to have a microscopic origin. Such variations seem more consistent with a model of microscopic critical current fluctuators, suggesting that both MRT and microresonator effects are present 12. If this is the case, it is important to identify which observed splittings are due to which mechanism.

Measured with sufficient resolution, the transition frequency avoided crossings due to MRT should have distinctive characteristics. When driving $0 \rightarrow 1$ transitions, a splitting in the $|0\rangle_{L}$ branch should produce crossings like that shown in Fig. 3(a), whereas a splitting in the $|1\rangle_{L}$ branch should produce crossings like that shown in Fig. 3(b). The observed shapes may be strongly dependent upon the experimental measurement technique. Bias noise could smear out the splittings in the horizon-
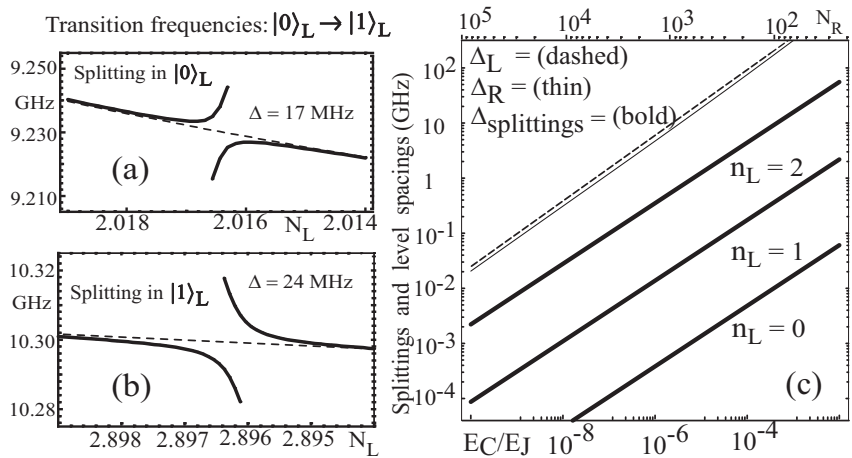

FIG. 3: (a) The distinctive shapes of avoided crossings in the measured transition frequencies for splittings in the lower branch. (b) The avoided crossing transition shape for splittings in the upper branch. (c) The figure shows that $\Delta_{R} \approx \Delta_{L}$ over an extremely large range of double-well circuit parameters. Bold lines show the splitting magnitudes $\Delta$ along the $n_{L}=0,1$, and 2 left well energy branches, with $\beta=4.5$, $N_{L}=3$, and $I_{c}=10 \mu \mathrm{A}$.

tal direction. For splittings in the lower energy branch [Fig. 3(a)] this would leave a distinct frequency gap, givings observed splittings horizontally smeared appearances like those observed in 1, 2]. In contrast, it is unclear if splittings in the upper branch [Fig. 3(b)] are consistent with observation. Improved experimental resolution that revealed these distinctive avoided-crossing shapes would be compelling evidence for MRT. Han et al. have explored other complexities that arise when measuring systems that exhibit MRT 7].

We derive an analytic expression for the energy splitting between pair-wise degenerate left and right states in an asymmetric double well in the WKB approximation 13. This yields the splitting formula

$$
\Delta=\sqrt{\frac{2 \Delta_{L} \Delta_{R}\left(n_{L}+\frac{1}{2}\right)^{n_{L}+1 / 2}\left(m_{R}+\frac{1}{2}\right)^{m_{R}+1 / 2}}{\pi n_{L} ! m_{R} ! e^{n_{L}+m_{R}+1}}} e^{-S},
$$

where $S=\int_{\gamma_{1}}^{\gamma_{2}} \sqrt{2 m[E-V(\gamma)]} d \gamma, m=C\left(\Phi_{0} / 2 \pi\right)^{2}$, $\gamma_{1,2}$ are the classical turning points for the barrier given by $V\left(\gamma_{1,2}\right)=E_{n_{L}}$, and $\Delta_{L} \simeq \hbar \omega_{L}, \Delta_{R} \simeq \hbar \omega_{R}$ are the left and right well level spacings at energy $E_{n_{L}}$. For deep right wells, Eq. (5) becomes independent of $m_{R}$. In this limit, together with the cubic approximation accurate for shallow left wells, the splittings are approximately

$$
\Delta \simeq \sqrt{\frac{2^{1 / 2} \Delta_{L} \Delta_{R}}{n_{L} ! \pi^{3 / 2}}}\left(432 N_{L}\right)^{\left(n_{L}+1 / 2\right) / 2} e^{-18 N_{L} / 5} .
$$

For the right well level spacing we use the WKB estimate $\Delta_{R}=2 \pi \hbar / T_{c l}$ [14], where $T_{c l}$ is the classical period of right-well oscillations with energy $E_{n_{L}}$. Splittings calculated from Eq. (6) are shown as solid lines in Fig. 2(d). The agreement with the exact splittings (solid points) is excellent for lower lying states, and surprisingly good for 
the excited states. Note that the tunnel splitting formula in Eq. (6) predicts splittings exponentially larger than continuum tunneling rates: $\Delta_{\text {splitting }} / \Gamma_{\text {tunneling }} \sim$ $\exp \left(18 N_{L} / 5\right)$, making MRT effects important even when continuum tunneling is negligible.

We have compared MRT splittings with Eq. (6) for a number of numerical examples with $N_{R} \sim 100-1000$, but in principle one can fabricate circuits with many thousands of right-well states. The WKB formula for the splittings and level spacings allows analysis of circuit parameters for very deep right wells where numerical treatment is impractical. Figure 3(c) shows $\hbar \omega_{L} \simeq \Delta_{L}$ (dashed line) and $\Delta_{R}=2 \pi \hbar / T_{c l}$ (thin-solid line) versus the ratio $E_{C} / E_{J}$ for $I_{c}=10 \mu \mathrm{A}, N_{L}=3$, and $\beta=4.5$ just below the $\beta$ threshold where the potential develops three wells. (For the circuit parameters in Fig. 2 and 2], $E_{C} / E_{J} \sim 10^{-4}-10^{-6}$.) The value of $I_{c}$ determines the frequency scale on the left of Fig. 3(c) but leaves the relative positions of the plotted lines essentially unchanged. The top axis shows $N_{R}$ from the harmonic oscillator approximation. Observe that, perhaps unexpectedly, $\Delta_{R} \approx \Delta_{L}$ even for extremely asymmetric double wells. The bold-solid lines show the WKB splitting $\Delta$ when $n_{L}=0,1$, and 2 . The validity condition for MRT $\Delta \ll \Delta_{R, L}$ is satisfied over a large range of circuit parameters, and for $N_{R} \sim 10^{5}$ and greater.

Dissipation suppresses resonant tunneling when $\Gamma_{R} \gtrsim$ $\Delta_{R}$, where $\Gamma_{R} \simeq N_{R} \hbar / T_{1}$ is the width of excited right well states, and $T_{1}$ is the dissipation time for $|1\rangle_{R} \rightarrow$ $|0\rangle_{R}$ [15, 16]. Using the WKB expression for $\Delta_{R}$, we find the condition $N_{R} \lesssim \omega_{L} T_{1}$ for observing MRT. For a phase qubit with $\omega_{L} / 2 \pi \sim 10 \mathrm{GHz}$ and $T_{1} \sim 10-100$ ns, resonant tunneling should be detectable as long as $N_{R} \lesssim 600-6000$ states. For the circuit parameters in Fig. $2 N_{R} \sim 600-700$ and for those in $[2] N_{R} \sim 150-300$, with a measured $T_{1} \simeq 25 \mathrm{~ns}$. Thus, we do not believe that dissipation will remove the effects of MRT. If the intrinsic dissipation is actually much smaller so that $\Gamma \lesssim \Delta 16$, it should be possible to observe coherent oscillations [17].

In conclusion, we show that significant MRT effects should be present for extremely asymmetric double well phase qubits, and thus MRT should be taken into account in the important effort to fully characterize microresonators or other splittings mechanisms. Our analysis provides tools and can guide experiments to help distinguish between three main possibilities: (1) Both MRT and microresonators are present, (2) MRT effects explain all the observational data, and (3) MRT is entirely absent. We believe that (1) is most likely; however, due to the complexity of effects from MRT, further experiments and detailed modeling are necessary to definitively rule out (2) and (3). Finally, our Letter provides general tools for exploring the quantum mechanics of extremely asymmetric double-well systems.

We thank R. Simmonds and J. Martinis for useful comments. This work was supported by the NSA, the DCI
Postdoctoral Research Program, the NSF QUBIC program, DOE grant DE-FG02-96ER40949, and the University of Maryland's Center for Superconductivity Research.

* electronic address: philipj@physics.umd.edu

$\dagger$ electronic address: fstrauch@physics.umd.edu

[1] R.W. Simmonds et al., Phys. Rev. Lett 93, 077003 (2004).

[2] K.B. Cooper et al., Phys. Rev. Lett. 93, 180401 (2004).

[3] D.J. Van Harlingen et al., Phys. Rev. B 70, 064517 (2004); F. Meier and D. Loss, cond-mat/0408594 (2004).

[4] J.M. Martinis, S. Nam, J. Aumentado, and C. Urbina, Phys. Rev. Lett. 89, 117901 (2002); Y. Yu et al., Science 296, 889 (2002); A. J. Berkley et al., ibid. 300, 1548 (2003); F.W. Strauch et al., Phys. Rev. Lett. 91167005 (2003); H. Xu et al., ibid. 94, 027003 (2005).

[5] T. Yamamoto et al., Nature (London) 425, 941 (2003); Yu. A. Pashkin et al., ibid. 421, 823 (2003); A. Wallraff et al., ibid. 431, 162 (2004); D. Vion et al., Science 296, 886 (2002).

6] C. H. van der Wal et al., Science 290, 773 (2000); I. Chiorescu, Y. Nakamura, C.J.P.M. Harmans, and J.E. Mooij, Science 299, 1869 (2003); A. Izmalkov et al., Phys. Rev. Lett. 93, 037003 (2004); Y. Yu et al., ibid. 92, 117904 (2004); L. Tian, S. Lloyd, and T.P. Orlando, Phys. Rev. B 67, 220505 (2003).

[7] R. Rouse, S. Han and J.E. Lukens, Phys. Rev. Lett. 75, 1614 (1995); S. Han, R. Rouse, and J.E. Lukens, ibid. 84, 1300 (2000); J.R. Friedman et al., Nature (London) 406, 43 (2000); D.V. Averin, J.R. Friedman, and J.E. Lukens, Phys. Rev. B 62, 11802 (2000).

[8] D.S. Crankshaw et al., Phys. Rev. B 69, 144518 (2004).

[9] P. Bertet et al., cond-mat/0412485 (2004); B.L.T. Plourde et al., cond-mat/0501679 (2005).

[10] C.C. Marston and G.G. Balint-Kurti, J. Chem. Phys. 91, 3571 (1989)

[11] Fig. 1(b) of [1] shows $N_{R} \approx 2500$.

[12] R. Simmonds and J. Martinis, private communication.

[13] We derived Eq. (5) from Herring's formula assuming a WKB wavefunction under the barrier and matching onto excited harmonic oscillator states in the two wells. This method is described in A. Garg, Am. J. Phys. 68, 430 (2000). See also J.M. Schmidt, A.N. Cleland, and J. Clarke, Phys. Rev. B 43, 229 (1991).

[14] L. D. Landau and E. M. Lifshitz, Quantum mechanics, volume III, Pergamon Press (London) 1965.

[15] A.O. Caldeira and A.J. Leggett, Ann. Phys. (New York) 149, 374 (1983).

[16] A. Garg, Phys. Rev. B 51, 15161 (1995).

[17] It is interesting to speculate that unexpectedly short dissipation times of $\sim 25 \mathrm{~ns}$ in [2] result from damping of excited right well states, such that $T_{\text {measured }} \sim T_{1} / N_{R}$. This would imply a $T_{1}$ of a few $\mu$ s, consistent with the expected relaxation times (see footnote [11] in [2]). The existence of tunnel splittings could also be probed by tuning $T_{1}$ through adjustable coupling to adjacent circuit elements. 